\title{
Novel mutation in exon 56 of the dystrophin gene in a child with Duchenne muscular dystrophy
}

\author{
JIAN-FANG $\mathrm{ZHU}^{1 *}$, HUI-HUI LIU ${ }^{2 *}$, TAO ZHOU $^{3}$ and LI TIAN ${ }^{4}$ \\ ${ }^{1}$ Central Laboratory of Union Hospital, Huazhong University of Science and Technology, Wuhan, Hubei 430022; \\ ${ }^{2}$ Department of Anesthesiology, The First Affiliated Hospital, Guangzhou University of Traditional Chinese Medicine, \\ Guangzhou, Guangdong 510405; Departments of ${ }^{3}$ Otolaryngology and ${ }^{4}$ Pediatrics, Union Hospital, \\ Huazhong University of Science and Technology, Wuhan, Hubei 430022, P.R. China
}

Received June 10, 2013; Accepted September 12, 2013

DOI: $10.3892 /$ ijmm.2013.1498

\begin{abstract}
Duchenne type muscular dystrophy (DMD) is an allelic $\mathrm{X}$-linked recessive disorder caused by mutations in the gene encoding dystrophin. Genotype analysis has shown that deletion mutations account for approximately $65 \%$ of all cases, and $5-10 \%$ are duplications, while the remaining $30 \%$ of affected individuals may have smaller mutations, including point mutations, small deletions or small insertions. In this study, we present the case of a 4-year-old boy with typical clinical features of DMD, who developed normally until the age of 2 . However, at age 3 he presented his first symptom, a tendency to fall, had difficulty in rising from the floor and in walking on his toes. At age 4 he had a waddling gait and could no longer climb stairs. A physical examination revealed proximal muscle weakness, calf hypertrophy, deep tendon hyporflexia and a positive Gower's sign. To identify the disease-causing gene in the proband, all coding regions (exons 1-79) of the dystrophin gene were PCR-amplified and sequenced. A novel duplication (c.8284dupA) in exon 56 of the dystrophin gene was identified, which was predicted to generate a frameshift mutation and create a premature termination codon (p.Ile2762Asnfs*10). This mutation was further confirmed by single-strand confor-
\end{abstract}

Correspondence to: Dr Tao Zhou, Department of Otolaryngology, Union Hospital, Huazhong University of Science and Technology, 1277 Jiefang Avenue, Wuhan, Hubei 430022, P.R. China

E-mail: entzt2013@sina.cn

Dr Li Tian, Department of Pediatrics, Union Hospital, Huazhong University of Science and Technology, 1277 Jiefang Avenue, Wuhan, Hubei 430022, P.R. China

E-mail: doctortianli@-yahoo.com.cn

*Contributed equally

Abbreviations: CR, cysteine-rich domain; CT, C-terminal domain; DGC, dystrophin-glycoprotein complex; DMD, Duchenne muscular dystrophy; MD, muscular dystrophy; SSCP, single-strand conformation polymorphism analysis

Key words: Duchenne muscular dystrophy, dystrophin gene, duplication, inherit, mutation mation polymorphism (SSCP) analysis, which revealed an extra band found in exon 56 of the dystrophin in the proband; however, this was not present in his family members or in the 100 matched normal controls. The data presented in this study may aid in expanding the spectrum of mutations causing DMD. To our knowledge, we demonstrate for the first time that a small duplication mutation can cause severe DMD.

\section{Introduction}

Muscular dystrophy (MD) refers to a genetically heterogeneous group of degenerative muscle disorders that are characterized by the progressive loss of muscle strength and integrity (1), and the most common and severe type of MD is Duchenne muscular dystrophy (DMD; MIM\#310200), which accounts for more than half of all MD cases (2-4) and affects approximately 1 in 3,500 live male newborns $(5,6)$. This disease is associated with continuous cycles of muscle cell regeneration and degeneration, ultimately resulting in the failure of muscle regeneration; the muscle is substituted by fat and connective tissue. Clinical symptoms include constant falling, waddling and out-turned knees, which appear as early as the age of 2 . Pathological studies have shown that DMD is triggered primarily by the decreased function of a vital muscle protein known as dystrophin, which is a long, rod-shaped molecule composed of 3,685 amino acid residues. It contains 4 distinct domains: an N-terminal actin binding domain, a central rod domain containing a second actin binding domain, a cysteinerich (CR) domain and a C-terminal (CT) domain (7).

In 1987, the dystrophin gene was cloned by Koenig et al $(8,9)$ approximately 150 years after the discovery of DMD, which is the largest human gene, which spans $>3,000 \mathrm{~kb}$ on the $\mathrm{X}$-chromosome and encodes a 14-kb transcript that consists of 79 exons, 78 introns and 8 promoters (10-13). Genotype analysis has revealed that DMD is a common X-linked recessive neuromuscular disorder caused by mutations of this gene inherited through the mothers who are carriers or that arise from germ line mosaicism. To date, there are 2 types of identified mutations of the dystrophin gene: deletions, which constitute approximately $60 \%$ of the mutations and generally involve the region of a major 3'-hotspot and a minor 5'-hotspot, exons 1-11 and 41-54 regions and non-deletions, which constitute $40 \%$ of 
the mutations, including point mutations, small deletions and insertions of the dystrophin gene $(14,15)$.

In this study, we analyzed the coding sequences of the dystrophin gene in a Chinese family and identified a novel duplication mutation in exon 56 of the dystrophin gene that causes DMD, expanding the spectrum of mutations causing DMD. To our knowledge, we are the first to demonstrate that a novel duplication of a single base can cause severe DMD.

\section{Materials and methods}

Patients. The study participants were identified and enrolled at Huazhong University of Science and Technology Union Hospital, Wuhan, China. Informed consent was obtained from the participants in accordance with the study protocols approved by the Ethics Committee of Huazhong University of Science and Technology. Fourteen family members, including 9 males and 5 females participated in this study (Fig. 1A). Detailed records of their medical history, physical examinations and histopathological analysis of muscle tissue were obtained. The diagnosis of DMD was made on the basis of symptoms, physical signs and blood creatine kinase (CK) levels. Among all the family members, only the proband presented the clinical criteria of the DMD phenotype.

Direct DNA sequencing analyses. As previously described (16), venous blood $(5 \mathrm{ml})$ was collected from the participants, and total human genomic DNA was isolated using the DNA Isolation kit for Mammalian Blood (Roche Diagnostics, Indianapolis, IN, USA). Considering that the dystrophin gene mutation is the genetic factor in DMD, we carried out mutation screening in the dystrophin gene directly without performing linkage analysis. The entire 79 exons and the exon-intron boundaries of the dystrophin gene of the proband were amplified by polymerase chain reaction (PCR). Primers were designed to amplify DNA fragments to span all 79 exons (range, of 200-800 bp; primers not shown). The nucleotide sequence of the DMD gene was obtained from GenBank (sequences of the primers are available upon request). Each amplicon was designed for an optimal size, with the exon centered within the amplicon. As a result, a total of 79 amplicons were used to sequence the coding region of the gene. Briefly, for PCR amplification, amplification was performed in a PTC-200 thermal cycler (MJ Research Inc., Waterdown, MA, USA) in a $25-\mu 1$ reaction mixture containing $1.5 \mathrm{mM} \mathrm{MgCl} 2,0.2 \mathrm{mM}$ of dNTP (Qiagen, Hilden, Germany), $0.5 \mu \mathrm{M}$ primers, $1 \mathrm{U}$ of TaqDNA polymerase (Qiagen) and $50 \mathrm{ng}$ of genomic DNA. PCR was performed as follows: an initial denaturation step was carried out for $5 \mathrm{~min}$ at $94^{\circ} \mathrm{C}, 9$ cycles of $45 \mathrm{sec}$ at $94^{\circ} \mathrm{C}, 45 \mathrm{sec}$ at $61.5^{\circ} \mathrm{C}$ and $45 \mathrm{sec}$ at $72^{\circ} \mathrm{C}$, followed by the same 29 cycles with a separate annealing temperature at $55^{\circ} \mathrm{C}$. Direct bidirectional resequencing of all PCR-amplified products was performed using the BigDye Terminator Cycle Sequencing v3.1 kit (Applied Biosystems, Foster City, CA, USA) and electrophoresed on an ABI PRISM 3730 Genetic Analyzer (Applied Biosystems). Sequencing results from the subjects and dystrophin gene consensus sequences from GenBank (GenBank accession no. M18533) were compared using BLAST analysis. Mutation description followed the nomenclature recommended by the Human Genomic Variation Society. Resequencing of the mutated exon 56 of the dystro- phin gene was performed on the 13 family members and the 100 unrelated controls and the primers used were as follows: forward, GGCACTGGGGTACACTTTATCATAGAA; and reverse, GCTGCACTCCTCATTTAAATTCACTCT.

Single-strand conformation polymorphism (SSCP) analysis. To confirm the mutation and determine whether the mutation co-segregates with the disease in the family, the novel variation detected in exon 56 of the dystrophin gene was further evaluated in the 14 available family members, as well as the normal control subjects using SSCP analysis, as previously described (17). Briefly, as mentioned above, PCR amplification was performed on exon 56 of the dystrophin gene and the primers used were as follows: forward, GAAAAGGGATTTGAGATGTA; and reverse, GTGCTAAGACAATGAGGAAA, and an initial denaturation annealing temperature at $60^{\circ} \mathrm{C}$ and a separate annealing temperature at $53^{\circ} \mathrm{C}$. Subsequently, $2 \mu 1$ of undigested PCR products were mixed with $4 \mu \mathrm{l}$ of the degenerating loading buffer, denatured at $95^{\circ} \mathrm{C}$ for $10 \mathrm{~min}$ and immediately placed on ice; they were then loaded on $6 \%$ polyacrylamide gels and the DNA samples were separated by electrophoresis overnight at $150 \mathrm{~V}$. The DNA bands were visualized by silver staining.

\section{Results}

Pedigree and clinical features of the family. The patient was a 4-year-old boy of Chinese origin, born after a normal term pregnancy. He developed normally until the age of 2. Family history was negative and his parents were not consanguineous. He began to walk when at 18 months of age. However, at age 3 he presented his first symptom, a tendency to fall, and had difficulty in rising from the floor and in walking on his toes. At age 4 he had a waddling gait and could no longer climb stairs. A physical examination revealed proximal muscle weakness, calf hypertrophy, a mild weakness of the limb-girdle muscles, deep tendon hyporflexia, hyperlordosis and a positive Gower's sign. Serum muscle enzyme concentrations were markedly increased to $33,400 \mathrm{U} / 1$ (normal: $37-174 \mathrm{U} / 1$ ). His muscle biopsy specimen revealed dystrophic features with a wide variation in fiber size, including fiber hypertrophy, degeneration, atrophy and an increase in endomysial connective tissue (Fig. 1C). However, all the clinical features of the proband were not discovered in the other members of his family, particularly his mother.

Mutation analysis. To identify the molecular basis of DMD in the proband, exons 1-79 of the dystrophin gene were amplified by PCR. By direct bidirectional sequencing of the PCR products in the proband, a duplication at position 8284 (c.8284dupA) in exon 56 of the dystrophin gene (Fig. 2A) was revealed; this was confirmed by repeating the experiment. We did not find any other mutation or polymorphism in the exons of the dystrophin gene. Since DMD is a common X-linked recessive neuromuscular disorder, direct sequence analysis of DNA from his parents and brother was also performed on exon 56 of the dystrophin gene. However, the sequence was the same as that of the normal one (Fig. 2B).

Analysis of the changes in dystrophin protein sequences after mutation. A duplication (c.8284dupA) in the dystrophin gene 

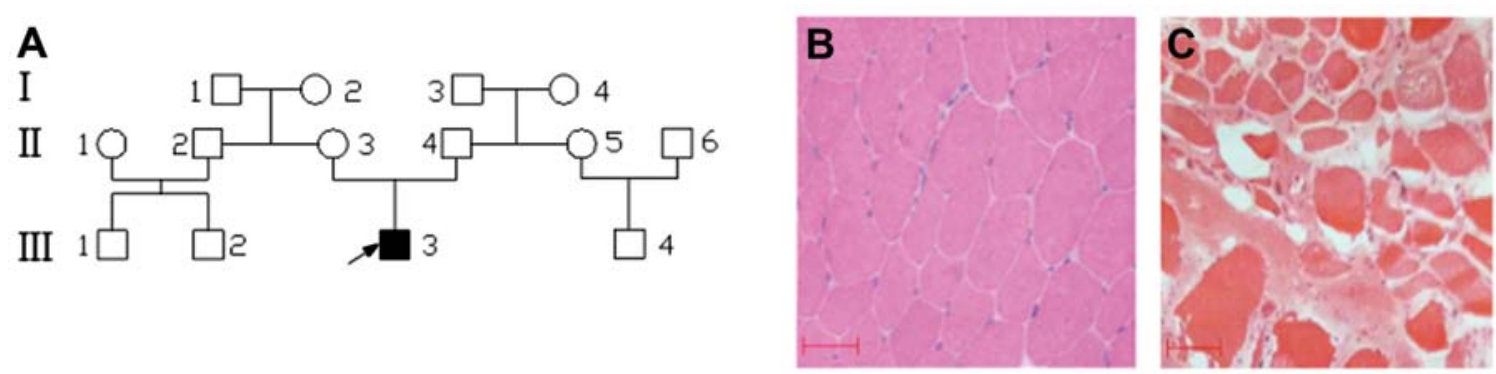

Figure 1. (A) Pedigree chart of a Chinese Han family who has a child with Duchenne muscular dystrophy (DMD), which is indicated by filled symbols. The arrowhead indicates the proband (III3). (B and C) Transverse sections of skeletal muscle shown by hematoxylin and eosin staining (H\&E). (B) Normal polygonal myofibers with peripheral nuclei and uniform diameter in a panel from his father (II4). (C) Polygonal myofibers with a wide variation in fiber size, including fiber hypertrophy, degeneration, atrophy and an increase in endomysial connective tissue from the proband (III4). Scale bar, 50 microns.

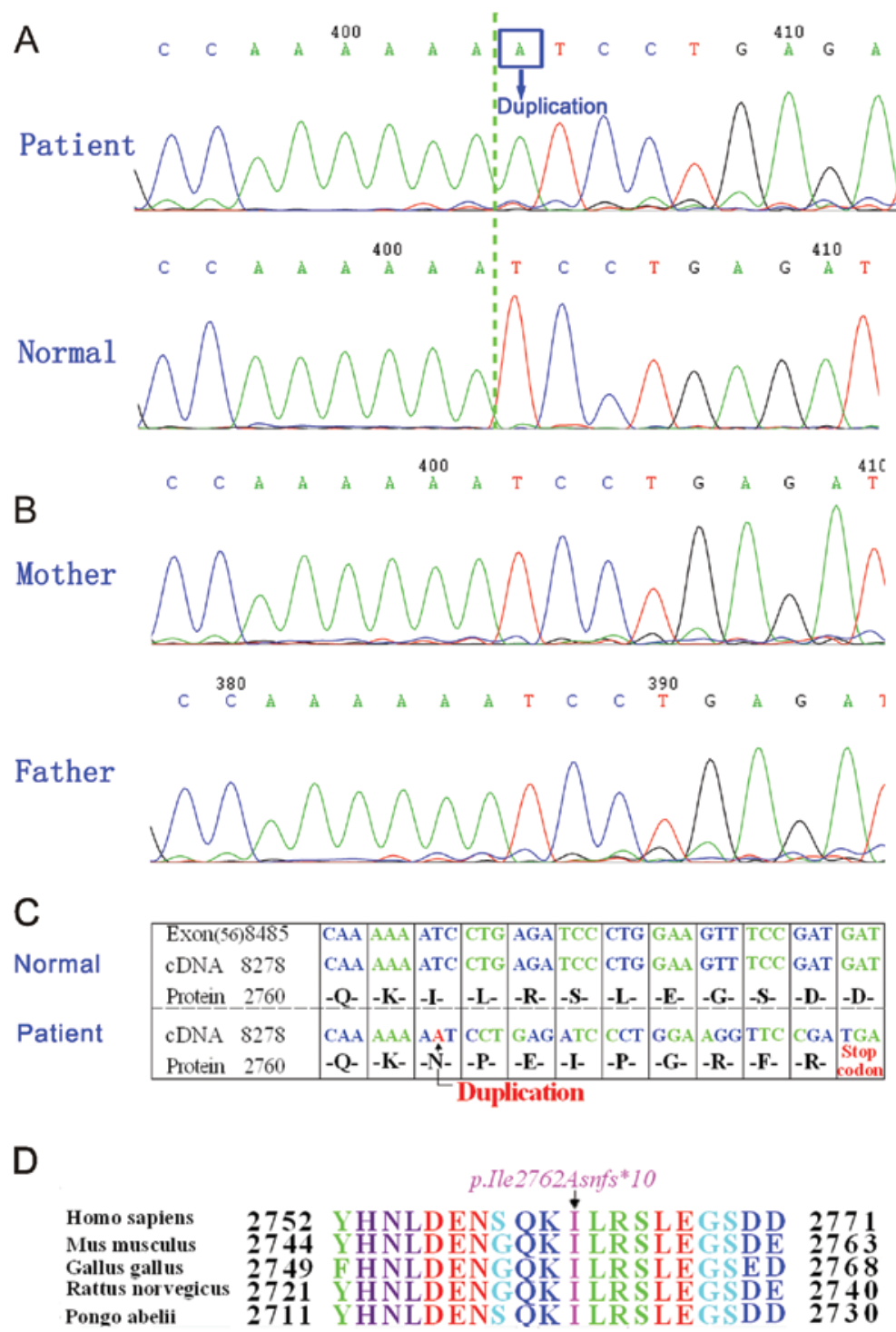

Figure 2. Analysis of the mutation of the dystrophin gene in the family and changes in dystrophin protein sequences after mutation. (A) Sequencing chromatograms of the proband from the family and an unrelated control. The results show a duplication mutation (c.8284dupA) in exon 56 of the dystrophin gene in the proband. (B) Sequencing chromatograms of his parents. The results show a normal DNA sequence without this mutation. (C) A duplication mutation causes a termination codon TGA that results in a truncated product of 2,770 amino acid residues (p.Ile2762Asnfs*10). (D) CLUSTALW alignment of the dystrophin protein shows that isoleucine (I) residue at position 2762 is highly conserved across humans, mice, chickens, rats and orangutans.

causes a termination codon TGA that results in a truncated product of 2,770 amino acid residues (p.Ile2762Asnfs*10) (Fig. 2C) instead of the wild-type length of 3,685 amino acid residues. Ile2762 is highly conserved among humans (Homo sapiens), mice (Mus musculus), chickens (Gallus gallus), rats (Rattus norvegicus) and orangutans (Pongo abelii) (Fig. 2D). 


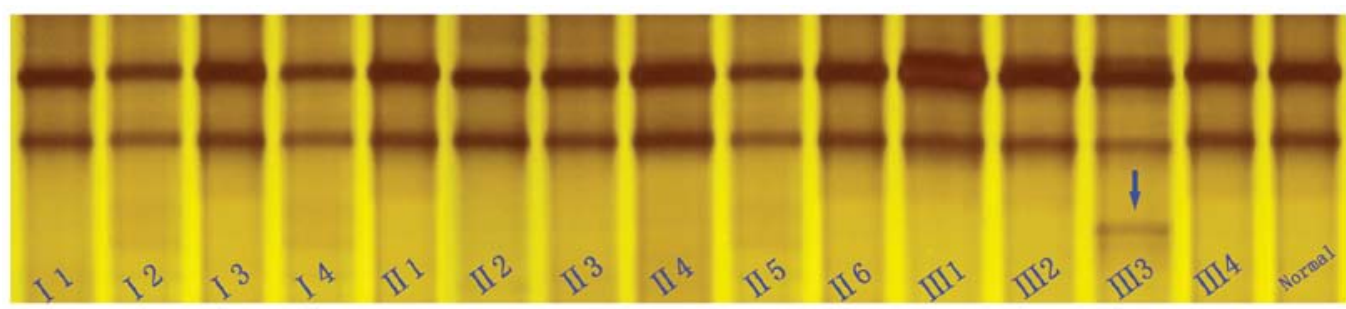

Figure 3. Single-strand conformation polymorphism (SSCP) analysis of the mutation in exon 56 of the dystrophin gene. The proband has one more band than the normal family members. The arrow indicates the extra band (III3).

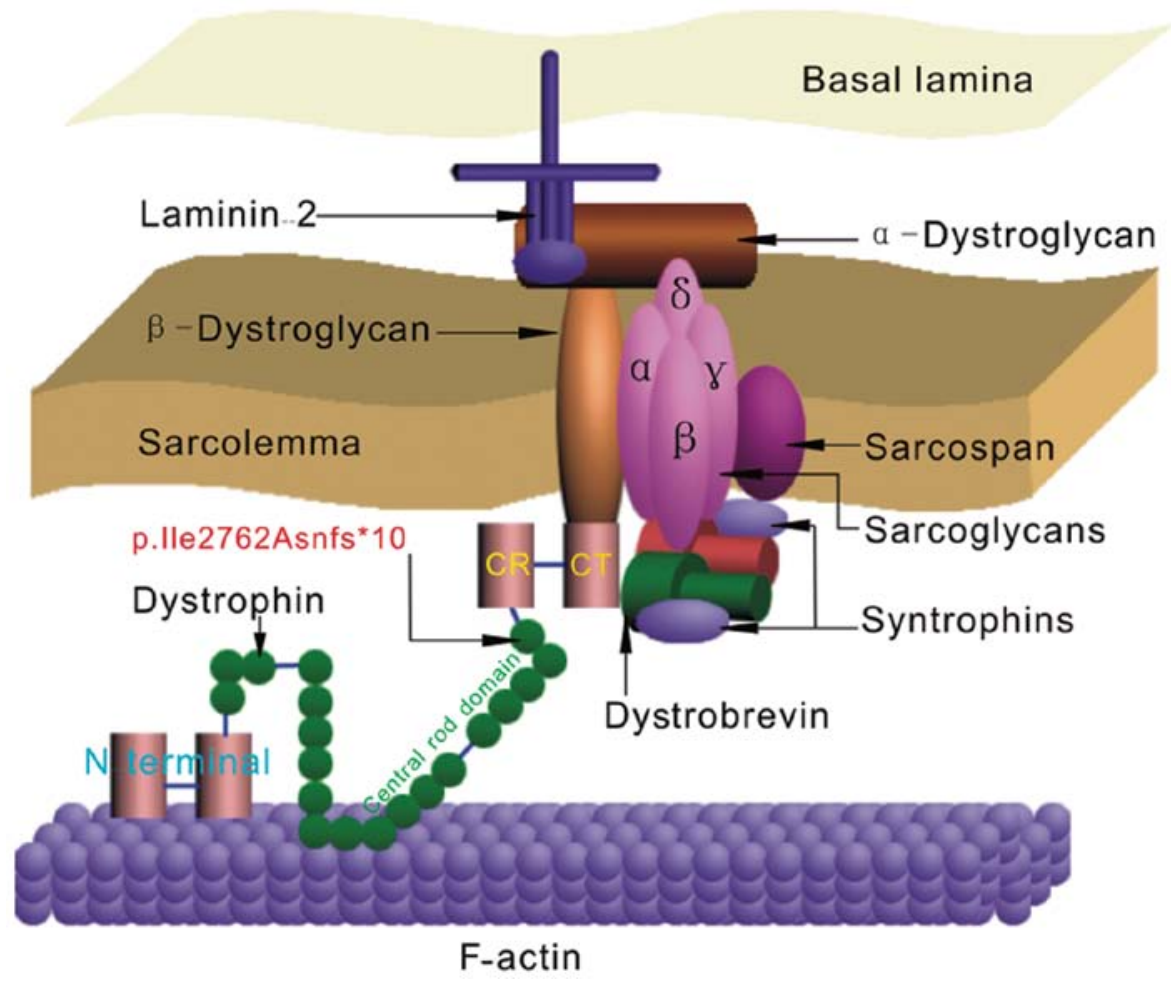

Figure 4. Illustration of the molecular structure of the dystrophin-glycoprotein complex (DGC) (19,25). Dystrophin includes 4 distinct domains: an N-terminal actin binding domain, a central rod domain containing a second actin binding domain, a cysteine-rich (CR) domain and a C-terminal (CT) domain. In this study, a duplication (c.8284dupA) results in a frameshift mutation and a premature termination 10 codons downstream (p.Ile2762Asnfs*10).

SSCP analysis. To confirm the mutation and test whether the mutation co-segregates with the disease in the family, the novel variation detected in exon 56 of the dystrophin gene was further evaluated in the 14 available family members, as well as in the normal control subjects using SSCP analysis (Fig. 3). The SSCP results revealed that an extra band was found in exon 56 of the dystrophin gene. However, the DNA samples from the 100 normal males and all the family members were also analyzed by SSCP, and the results revealed that the unaffected members of the family and the 100 normal Chinese Han controls did not carry this mutation. These results further suggest that this novel mutation (c.8284dupA, p.Ile2762Asnfs"10) of the dystrophin gene is not a rare polymorphism, but a causative mutation for DMD in the proband.

\section{Discussion}

The present study demonstrates that the newly identified mutation in exon 56 of the dystrophin gene (c.8284dupA) is associated with DMD in a child in a Chinese family, and that this mutation is a de novo mutation, but not an $\mathrm{X}$-linked recessive mode of inheritance from the mother. First, the fact that the clinical manifestations of proximal muscle weakness, calf hypertrophy, mild weakness of the limb-girdle muscles, deep tendon hyporflexia, hyperlordosis and a positive Gower's sign occurred only in the proband. The other members did not present these clinical features. Second, the genotype revealed a duplication at position 8284 (c.8284dupA) in exon 56 of the dystrophin gene, but the mother and brother had a normal genotype at this position. Furthermore, the fact that the mutation found in the proband was not detected in the 100 unrelated control subjects without DMD by SSCP excludes the possibility that this mutation is a polymorphism.

Full-length dystrophin is a large rod-shaped protein with a molecular weight of $427 \mathrm{kDa}$ that contains 3,685 amino acid (AA) residues and comprises 4 domains (Fig. 4), which include the actin-binding (N-terminal) domain (AA \#14-240, exons 2-8), the central rod domain (AA \#253-3040, exons 8-61) 
consisting of 24 spectrin repeats and 4 hinge regions, the CR domain (AA \#3080-3360, exons 62-69) and the CT domain (AA \#3361-3685, exons 69-79) (18-21). In this study, we find the newly identified mutation in exon 56 of the dystrophin gene (c.8284dupA), which results in a frameshift mutation and a premature termination 10 codons downstream (p.Ile2762Asnfs*10) by stop codon TGA, which is thus far the most frequent point mutation found in the DMD gene (10/47) (22). Due to this mutation, the entire CR and CT domain was lacking. In the context of a full-length dystrophin protein, the essential CR and CT domain was not retained; the protein is completely non-functional (23), which seems to cause severe symptoms (24). These results are consistent with the conclusion of Koenig et al $(8,9)$, who pointed out that the nucleotide sequence corresponding to the $\mathrm{CR}$ and $\mathrm{CT}$ domain was lacking in most DMD cases based on their dystrophin gene mutation analysis. Dystrophin is associated with the plasma membrane of cardiac and skeletal muscle (sarcolemma) and its main role in the sarcolemma is to interact with integral membrane proteins (sarcoglycan, dystroglycans, syntrophin and dystrobrevin complexes) that are assembled in the dystrophin-glycoprotein complex (DGC) through its CR and CT domains (Fig. 4) (25), including the last 54 amino acid residues of the rod to the CR domain (amino acid residues \#3026-3345) (26). Therefore, the lack of these domains in the dystrophin gene leads to destabilization and loss of the DGC (27). Thus, as long as the $\mathrm{CR}$ and $\mathrm{CT}$ are deleted, the binding between syntrophin and dystrobrevin is eliminated. Consequently, the function of the muscle is diminished.

In conclusion, although several point mutations in the dystrophin gene associated with DMD have been identified worldwide $(28,29)$, in this study, we identified a novel duplication in exon 56 of the dystrophin gene in a Chinese child with DMD. To our knowledge, this is the first report of Chinese DMD patients with a duplication in the region of exon 56 , which results in truncated dystrophin lacking the CR and CT. This finding expands the mutation spectrum of the dystrophin gene and may prove useful and valuable for genetic counseling and prenatal diagnosis in families with DMD.

\section{References}

1. Emery AE: The muscular dystrophies. Lancet 359: 687-695, 2002.

2. Hoffman EP, Brown RH and Kunkel LM: Dystrophin: The protein product of the Duchenne Muscular Dystrophy locus. Cell 51: 919-928, 1987.

3. Emery AE: Population frequencies of inherited neuromuscular disorders - a world survey. Neuromuscul Disord 1: 19-29, 1991.

4. Blake DJ, Weir A, Newey SE and Davies KE: Function and genetics of dystrophin and dystrophin-related proteins in muscle. Physiol Rev 82: 291-329, 2002.

5. van Essen AJ, Kneppers AL, van der Hout AH, et al: The clinical and molecular genetic approach to Duchenne and Becker muscular dystrophy: an updated protocol. J Med Genet 34: 805-812, 1997.

6. Sura T, Eu-ahsunthornwattana J, Pingsuthiwong S and Busabaratana M: Sensitivity and frequencies of dystrophin gene mutations in Thai DMD/BMD patients as detected by multiplex PCR. Dis Markers 25: 115-121, 2008.
7. Batchelor CL and Winder SJ: Sparks, signals and shock absorbers: How dystrophin loss causes muscular dystrophy. Trends Cell Biol 16: 198-205, 2006.

8. Koenig M, Hoffman EP, Bertelson CJ, et al: Complete cloning of the Duchenne muscular dystrophy (DMD) cDNA and preliminary genomic organization of the DMD gene in normal and affected individuals. Cell 50: 509-517, 1987.

9. Koenig M, Monaco AP and Kunkel LM: The complete sequence of dystrophin predicts a rod-shaped cytoskeleton protein. Cell 53: 219-228, 1988.

10. Ahn AH and Kunkel LM: The structural and functional diversity of dystrophin. Nat Genet 3: 283-291, 1993.

11. Nishio H, Takeshima Y, Narita N, et al: Identification of a novel first exon in the human dystrophin gene and of a new promoter located more than $500 \mathrm{~kb}$ upstream of the nearest known promoter. J Clin Invest 94: 1037-1042, 1994.

12. Magri F, Del Bo R, D'Angelo MG, et al: Clinical and molecular characterization of a cohort of patients with novel nucleotide alterations of the dystrophin gene detected by direct sequencing. BMC Med Genet 12: 37, 2011.

13. Dent KM, Dunn DM, von Niederhausern AC, et al: Improved molecular diagnosis of dystrophinopathies in an unselected clinical cohort. Am J Med Genet A 134: 295-298, 2005.

14. Tuffery-Giraud S, Chambert S, Demaille J and Claustres M: Point mutations in the dystrophin gene: evidence for frequent use of cryptic splice sites as a result of splicing defects. Hum Mutat 14: 359-368, 1999.

15. Wang Q, Li-Ling J, Lin C, et al: Characteristics of dystrophin gene mutations among Chinese patients as revealed by multiplex ligation-dependent probe amplification. Genet Test Mol Biomarkers 13: 23-30, 2009.

16. Cai F, Zhu J, Chen W, et al: A novel PAX6 mutation in a large Chinese family with aniridia and congenital cataract. Mol Vis 16: 1141-1145, 2010.

17. Wang Q, Shen J, Splawski I, et al: SCN5A mutations associated with an inherited cardiac arrhythmia, long QT syndrome. Cell 80: 805-811, 1995.

18. Sadoulet-Puccio HM and Kunkel LM: Dystrophin and its isoforms. Brain Pathol 6: 25-35, 1996.

19. Roberts RG: Dystrophins and dystrobrevins. Genome Biol 2: REVIEWS3006, 2001.

20. Davies KE, Tinsley JM and Blake DJ: Molecular analysis of Duchenne muscular dystrophy: past, present, and future. Ann NY Acad Sci 758: 287-296, 1995.

21. Ozawa E: Our trails and trials in the subsarcolemmal cytoskeleton network and muscular dystrophy researches in the dystrophin era. Proc Jpn Acad Ser B Phys Biol Sci 86: 798-821, 2010.

22. Wang J, Wang W, Li R, et al: The diploid genome sequence of an Asian individual. Nature 456: 60-65, 2008.

23. Nishida A, Kataoka N, Takeshima Y, et al: Chemical treatment enhances skipping of a mutated exon in the dystrophin gene. Nat Commun 2: 308, 2011.

24. Hall N: Advanced sequencing technologies and their wider impact in microbiology. J Exp Biol 210: 1518-1525, 2007.

25. Singh SM, Kongari N, Cabello-Villegas J and Mallela KM: Missense mutations in dystrophin that trigger muscular dystrophy decrease protein stability and lead to cross-beta aggregates. Proc Natl Acad Sci USA 107: 15069-15074, 2010.

26. Ishikawa-Sakurai M, Yoshida M, Imamura M, et al: $\mathrm{ZZ}$ domain is essentially required for the physiological binding of dystrophin and utrophin to beta-dystroglycan. Hum Mol Genet 13: 693-702, 2004.

27. Kemaladewi DU, Hoogaars WM, van Heiningen SH, et al: Dual exons kipping in myostatin and dystrophin for Duchenne muscular dystrophy. BMC Med Genomics 4: 36, 2011.

28. Hwa HL, Chang YY, Huang CH, et al: Small mutations of the DMD gene in Taiwanese families. J Formos Med Assoc 107: 463-469, 2008

29. Sitnik R, Campiotto $S$, Vainzof M, et al: Novel point mutations in the dystrophin gene. Hum Mutat 10: 217-222, 1997. 